\title{
DYNAMICAL ESTIMATION OF KEY CARDIAC-RESPIRATORY VARIABLES BY USING COMMERCIALIZED WEARABLE SENSORS
}

\author{
Kai Cao ${ }^{1}$, Lin $\mathrm{Ye}^{1}$, Hamzah.M.Alqudah ${ }^{1}$, Jan Szymanski ${ }^{1}$, Jing Zhou ${ }^{2}$, and Steven W. Su ${ }^{1}$ \\ ${ }^{1}$ Faculty of Engineering and Information Technology \\ University of Technology, Sydney \\ NSW 2007 Australia \\ Email: \{Kai.Cao-1, Lin.Ye-1, Hamzah.M.Alqudah\}@student.uts.edu.au, \\ \{Jan.Szymanski,Steven.Su\}@uts.edu.au \\ ${ }^{2}$ Department of Information and Telecommunication \\ Tongji University \\ Shanghai, China \\ Email: \{tjzj\}@tongji.edu.cn
}

\begin{abstract}
This paper investigates the estimation of key cardiacrespiratory variables (e.g., $V \mathrm{O}_{2}$ ) by using commercialised wearable sensors such as SensorTag and iPhone. The main aim of this study is to use inexpensive and user-friendly wearable sensors rather than expensive and cumbersome equipment (e.g., metabolic analyser). This study also aims to explore the possibility of using only embedded sensors of smart-phone to dynamically estimate oxygen consumption during moderate exercises. The major focus of this research is the modelling of the linear dynamic component. In order to capture the variance of linear dynamic characteristics (e.g., the time constant and steady state gain), we proposed a least square estimation algorithm equipped with automated equilibrium detection function. Finally, the effectiveness of the proposed approaches has been well demonstrated by experimental results.
\end{abstract}

\section{KEY WORDS}

VO2, Estimation, Modeling, Inertial sensor, IMU, Accelerometer

\section{Introduction}

In front of public health challenges, obesity has been increasing epidemic among adults and children[5]. According to the output of investigations in this area, obesity is linked with many other diseases, such as hypertension (HTN), type II diabetes mellitus, major cardiovascular (CV) diseases, etc[5]. To prevent obesity, leading a healthy life style is significant. Low-calorie diets are recommended and more importantly for most of people, low to moderate exercises are recommended[6]. If those cardiopulmonary data could be collected during moderate physical activities by some kind of healthy tracking devices, it would contribute to personal health management.
Most smart-phones have sensors (accelerometer, gyroscope and magnetometer) embedded, which provide a quite convenient way to generate various smart-phone based wearable devices, e.g., a pedometer, energy expenditure, sedentary reminder and so on[3][4][2]. However, the shortage of such devices is that only evaluating physical activates is not enough to monitor healthy status. It would be better that provides physiological data such as heart rate, pulse rate, etc. Heart rate $(\mathrm{HR})$ and oxygen uptake $\left(\mathrm{VO}_{2}\right)$ are the most commonly used parameters for assessment of metabolic demands[7]. In the previous study, D.B. Dill and etc., found a way to model steady state oxygen uptake[1]. The paper [8] and [7] gives a Hammerstein model identification for $\mathrm{VO}_{2}$ estimation. However, most devices for recording oxygen uptake are professional, expensive and cumbersome. Meanwhile, there are many factors that affect heart rate, e.g., emotional stress, medicine and so on. Hence, it is important to find an additional device that is able to monitor physical activities in order to minimise the effects of non-exercise factors.

The Inertial Measurement Unit (IMU) provides the possibility to monitor personal physical activities. With the base line of the heart rate and the intensity of workout, it is possible to predict some cardio-respiratory variables. It is evident that data has become a powerful and insightful tool providing health transparency to everyone. Hence, to find the relationship among $\mathrm{HR}, V \mathrm{O}_{2}$ and motion data is appreciable considering the implement of fitness tracker on cardiac-respiratory variables.

This paper aims to identify a dynamical estimation model for both onset and offset during moderate exercise. A method for the identification of linear dynamic model is developed for $\mathrm{HR}$ and $\mathrm{VO}_{2}$ estimation. The advantage of the proposed method is its function to automatically estimate the equilibriums for both onset and offset exercise.

The paper is arranged as follow: the equipment, ex- 


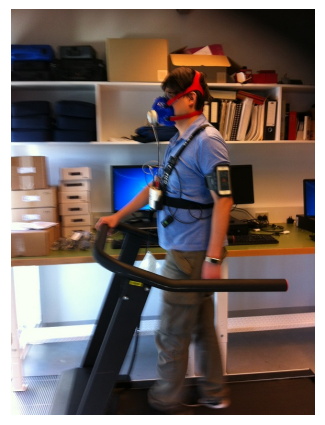

Figure 1: A volunteer in the experiment

periment setup and the technical details of data collection are given in Section 2. The modelling method is given in Section 3. Section 4 presents the experimental results and the related discussions. Section 5 will conclude this paper.

\section{Overview}

\subsection{Equipment}

In order to estimate oxygen uptake during locomotion, four kinds of equipment are required for the experiment. For breath analyzing system, mobile metabolic analyzer is used in this experiment. A portable metabolic system called COSMED $K 4 b^{2}$ was used. The portable system provides multiple parameters for breath by breath analysis, such as oxygen uptake $\left(\mathrm{VO}_{2}\right)$, carbon dioxide production $\left(\mathrm{VCO}_{2}\right)$, heart rate, and so on. The mainly used parameters were $V \mathrm{O}_{2}$ and $H R$. A heart rate monitor with an elastic chest strap which designed by Polar is attached to the metabolic system and the data of heart rate transmits to the $K 4 b^{2}$ via wireless port. A medical grade treadmill called Trackmaster FVX-325 was used. In order to control the speed of the treadmill, an application which precisely controls the running duration and speed was developed. The application was deployed on a Windows system with a LabView platform. For recording motion activities, two smart-phone and two SensorTags which include accelerometer, gyroscope and magnetometer were in employment. The SensorTag 2650 which is an IoT (Internet of Things) enabled device that designed by Texas Instruments is able to monitoring physical actives wirelessly via Internet. An application has been developed for SensorTag. Two iPhone were also used in this experiment for additional motion data recording.

\subsection{Experiment Setup}

In order to find the clues of the relationship among heart rate $(\mathrm{HR})$, oxygen uptake $\left(\mathrm{VO}_{2}\right)$ and motion data, one workout protocol is designed. It defines the duration and intensity of workout. For convenience, it called 2-5 protocol which sets initial walking speed at $2 \mathrm{~km} /$ hour (kilometers per hour) for 5 minutes and then fast walking for another 5 minutes at 5 $\mathrm{km} /$ hour for and finally slow down to $2 \mathrm{~km} /$ hour for another
10 minutes.

The $K 4 b^{2}$ requires at least 45 minutes warming up before conduction of an experiment. After warming up, it has to do three calibration including air calibration, reference gas calibration and last and most important gas delay calibration which ensures all the sensors in $K 4 b^{2}$ working simultaneously. Before running on the treadmill, an individual has to wear such sensors before walking on the treadmill. Firstly, put the heart rate sensor on chest below nipples. A conductive gel was applied on chest as well. Secondly, install the $K 4 b^{2}$ on the subject's chest and turn it on. Then put the SensorTag and the iPhone for motion data. Test and calibration the SensorTag with a recording computer. If everything is settled, then ask the volunteer standing up on treadmill. Then uses LabView to initial the process of experiment. During the test, make sure that data is streaming to computer. After the test, ask the volunteer to take a sit and then take off all the sensors. Four motion sensors were placed on a volunteer including SensorTags and iPhones that recorded accelerometer and gyroscope and magnetometer. The figure 1 shows basic setup.

\subsection{Participants}

There are five volunteers that helped the experiment under 2-5 protocol. The Table 1 shows five volunteers' personal information including age, height, weight and BMI.

Table 1: Mean and STD of Volunteers' physical information

\begin{tabular}{|l|l|l|l|l|}
\hline & Age & Height & Weight & BMI \\
\hline Mean & 28.6 & 176.2 & 78.8 & 25.3282 \\
\hline STD & 5.8566 & 5.0695 & 11.0318 & 3.0802 \\
\hline
\end{tabular}

\subsection{Data Acquisition and Preprocessing}

In this experiment, the SensorTag was used for monitoring physical exercise. In order to logging motion activities, IMU data including accelerometer, gyroscope and magnetometer was sent to a PC via Bluetooth (BLE). The PC is a Linux based system (Ubuntu 14.04) with python 2.7.

Cardio-respiratory parameters are not count by every second. In order to make those data logging as one second per recording, a quite convenient way as post data processing was proposed. It employs interpolate method to overcome the problem in MatLab. It can be seen the difference between original and interpolated data from figure 2 .

\section{Method}

\section{1 $\mathrm{HR}$ and $\mathrm{VO}_{2}$ Estimation}

During the treadmill exercise, two models are developed for $H R$-speed and $\mathrm{VO}_{2}$-speed. Let the $\mathrm{HR}$ and $\mathrm{VO}_{2}$ as output $Y(s)$ respectively, the relationship between user's $\mathrm{HR}, \mathrm{VO}_{2}$ 

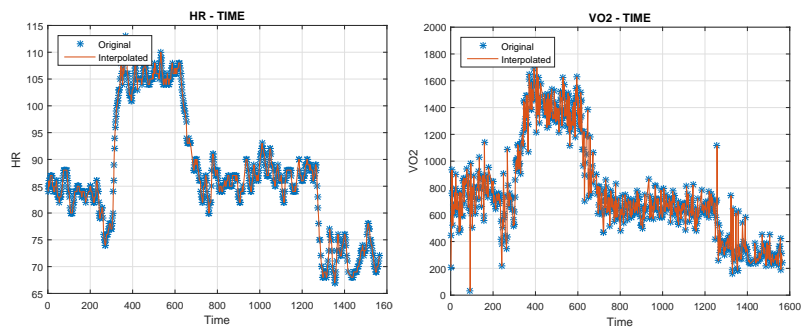

Figure 2: Original and interpolated data

and running speed $U(s)$ can be approximated by a first order system as:

$$
Y(s)=\frac{K U(s)}{T s+1},
$$

where $K$ represents the gain and $T$ represents the time constant.

To discretise the above system, it is adopting two different methods. First, the discrete time domain relationship for (1) which can be directly written as:

$$
T \frac{y[k]-y[k-1]}{T_{s}}+y[k]=K u[k],
$$

where $T_{s}$ is the sampling time.

In matrix form, we have:

$$
\left[\begin{array}{cc}
y[2]-y[1] & -T_{s} u[2] \\
y[3]-y[2] & -T_{s} u[3] \\
\vdots & \vdots \\
y[N]-y[N-1] & -T_{s} u[N]
\end{array}\right]\left[\begin{array}{c}
T \\
K
\end{array}\right]=\left[\begin{array}{c}
-T_{s} y[2] \\
-T_{s} y[3] \\
\vdots \\
-T_{s} y[N]
\end{array}\right] .
$$

Normally, matrix (3) can be solved with least square estimation (LSE) when all the inputs and outputs are available. More precisely, for modelling of $\mathrm{HR}$-speed and $\mathrm{VO}_{2}$-speed systems. The output should be the change of $H R$ (current $H R$ minus initial $H R$ ). For an accurate estimation, it is necessary to obtain the initial $H R$ to remove bias. In real world, it take times to obtain initial heart rate accurately and directly. In order to solve this problem, two extra variables are introduced as $a$ and $b$. Let rephrases $u[k]$ and $y[k]$ as:

$$
\begin{aligned}
& \tilde{u}[k]=u[k]+a, \\
& \tilde{y}[k]=y[k]+b .
\end{aligned}
$$

where $\tilde{u}$ and $\tilde{y}$ are measured values and $u$ and $y$ are true values. Eq.(3) can then be rewritten as:

$$
\begin{gathered}
{\left[\begin{array}{ccc}
\tilde{y}[2]-\tilde{y}[1] & -T_{s} \tilde{u}[2] & T_{s} \\
\tilde{y}[3]-\tilde{y}[2] & -T_{s} \tilde{u}[3] & T_{s} \\
\vdots & \vdots & \vdots \\
\tilde{y}[N]-\tilde{y}[N-1] & -T_{s} \tilde{u}[N] & T_{s}
\end{array}\right]} \\
=\left[\begin{array}{c}
T \\
K \\
a K+b
\end{array}\right] \\
=\left[\begin{array}{c}
-T_{s} y[2] \\
-T_{s} y[3] \\
\vdots \\
-T_{s} y[N]
\end{array}\right]
\end{gathered}
$$

If it assumes that $a=0$, then the initial $H R b$ can be solved by the equation above. For the second method, it uses ARMA model for the $\mathrm{HR}$-speed and $\mathrm{VO}_{2}$-speed systems. The first order system can be expressed as:

$$
y[n]=a y[n-1]+b u[n-1]+e[n],
$$

where $y[n]$ is output, $u[n]$ is input and $e[n]$ is error. Now, if assuming the 'initial' value is $h$, then it left:

$$
\begin{aligned}
y[n]+b & =\beta_{1}(y[n-1]+b)+\beta_{2} u[n-1] \\
& =\beta_{1} y[n-1]+\beta_{2} u[n-1]+\left(\beta_{1} b-b\right) .
\end{aligned}
$$

In matrix form, we have:

$$
\left[\begin{array}{ccc}
y[1] & u[1] & 1 \\
y[2] & u[2] & 1 \\
\vdots & \vdots & \vdots \\
y[n-1] & {[n-1]} & 1
\end{array}\right]\left[\begin{array}{c}
\beta_{1} \\
\beta_{2} \\
\beta_{1} b-b
\end{array}\right]=\left[\begin{array}{c}
y[2] \\
y[3] \\
\vdots \\
y[n]
\end{array}\right] .
$$

Eq.(9) can be solved by LS when the input $u[n]$ is not constant. Meanwhile, the initial $H R(\mathrm{~h})$ can be solve directly.

\section{Results of Experiments}

We applied the above method in real experimental data. Most of estimations are good but some of them might be affected by subject's adjusting breath. Most of them are not trained and some of them are overweighed. The method which automatically selected onset and offset points is the new feature of this approach provided. The table 2 shows statistic results of five participants for $\mathrm{VO}_{2}$ and HR's time constant, steady state gain, onset and offset point under mean and STD method.

It is also provided the result of HR/VO2's estimation for onset and offset. The figure 3 illustrates the HR/VO2 data during whole experiment. For better calculation without affecting estimation results, some of dataset were trimmed accordingly after interpolated. The figure 4 shows the onset and offset HR estimation during the experiment. The figure 5 shows the onset and offset $\mathrm{VO}_{2}$ estimation during the experiment.
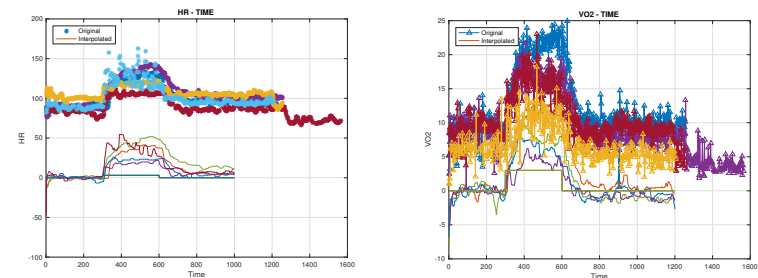

Figure 3: HR/VO2 original and estimation comparison for all 5 participants during full experiment process

\section{Conclusion}

The goal of this study is to investigate the estimation of dynamic cardiac-respiratory response of indoor treadmill 
Table 2: The mean and STD results of T, $\mathrm{K}$ and $\mathrm{H}$ for VO2 and HR in onset and offset

\begin{tabular}{|l|l|l|l|l|}
\hline \multirow{2}{*}{ Coefficients } & \multicolumn{2}{|c|}{ HR } & \multicolumn{2}{c|}{$V O_{2}$} \\
\cline { 2 - 5 } & MEAN & STD & MEAN & STD \\
\hline$T_{\text {onset }}$ & 11.455475 & 7.895173 & 28.903140 & 9.588534 \\
\hline$K_{\text {onset }}$ & 9.531647 & 3.332750 & 2.368064 & 1.043017 \\
\hline$H_{\text {onset }}$ & -92.214631 & 6.305837 & -8.944736 & 1.657193 \\
\hline$T_{\text {off set }}$ & 73.879023 & 26.442427 & 72.576536 & 31.899148 \\
\hline$K_{\text {offset }}$ & 8.957479 & 3.082858 & 2.615983 & 1.327074 \\
\hline$H_{\text {offset }}$ & -95.775417 & 7.231382 & -7.704993 & 1.824608 \\
\hline
\end{tabular}
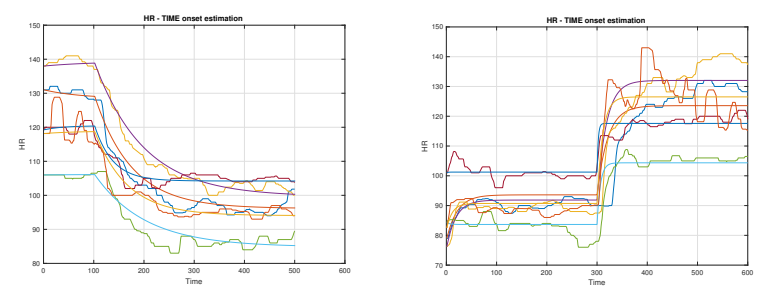

Figure 4: HR original and estimation comparison for all 5 participants during onset and offset
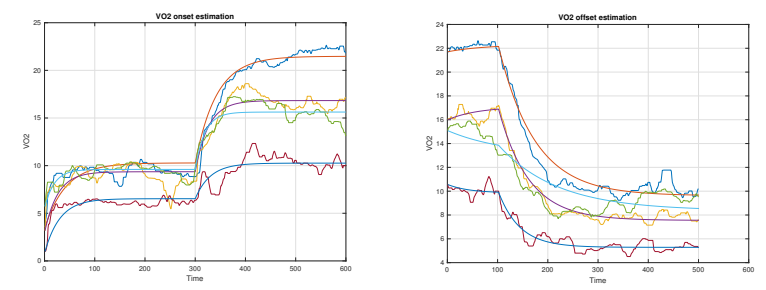

Figure 5: $V \mathrm{O}_{2}$ original and estimation comparison for all 5 participants during onset and offset

exercise and explore the possibly of only using commercially available wearable sensors and/or smartphone, in order to extend this estimation method from indoor exercise to outdoor. Different with existing studies, we proposed a Least Square based modelling algorithm to simultaneous estimate the dynamic characteristics of the model and its equilibrium variation between onset and offset exercises. The identified parameters, i.e., time constant, steady state gain, and equilibrium, indicate that these parameters have evident difference between onset and offset of exercises. This fact emphasises the necessity for the classification of onset and offset of exercises for dynamical estimation of exercise responses. Therefore, in the next step of this study, a more detailed analysis will be carried out for not only the identification of the relationship between walking/running speed and exercise frequency for individual exercisers between exerciser, based on motion data from smartphone, but also the classification of the patterns of onset and offset of exercises. It should be noted that the research results in this paper are mainly for the estimation of heart rate response and oxygen consumption rate. Another possible research topic is the extension to the estimation of other cardiac-respiratory variables, e.g., tidal volume, respiratory rate, and carbon dioxide, in free living condition by only using smartphone embedded sensors.

\section{References}

[1] D. DiLL, Oxygen used in horizontal and grade walking and running on the treadmill, Journal of Applied Physiology, 20 (1965), pp. 19-22.

[2] G. Geleijnse, A. VAN Halteren, And J. DiekHOFF, Towards a mobile application to create sedentary awareness, in CHI 2011: 29th ACM Conference on Human Factors in Computing Systems, Vancouver, Canada, 7-12 May 2011, ACM, 2011.

[3] P. Klasnja And W. PRATt, Healthcare in the pocket: mapping the space of mobile-phone health interventions, Journal of biomedical informatics, 45 (2012), pp. 184-198.

[4] P. V. Klasnja, S. Consolvo, D. W. McDonald, J. A. LANDAY, AND W. PRATT, Using mobile \& personal sensing technologies to support health behavior change in everyday life: lessons learned., in AMIA, Citeseer, 2009.

[5] C. J. Lavie, R. V. Milani, and H. O. Ventura, Obesity and cardiovascular disease: risk factor, paradox, and impact of weight loss, J Am Coll Cardiol, 53 (2009), pp. 1925-32.

[6] N. O. E. I. E. PANel ET AL., Clinical guidelines on the identification, evaluation, and treatment of overweight and obesity in adults, National Heart, Lung, and Blood Institute, 1998.

[7] S. W. Su, B. G. Celler, A. V. Savkin, H. T. Nguyen, T. M. Cheng, Y. Guo, And L. WAng, Transient and steady state estimation of human oxygen uptake based on noninvasive portable sensor measurements, Medical \& biological engineering \& computing, 47 (2009), pp. 1111-1117.

[8] S. W. Su, L. Wang, B. G. Celler, A. V. Savkin, AND Y. GUO, Identification and control for heart rate regulation during treadmill exercise, IEEE transactions on biomedical engineering, 54 (2007), pp. 1238-1246. 